\title{
Facial Recognition using Unsupervised Mode in Artificial Neural Network by Self-Organizing Map using MATLAB
}

\author{
Harsha Kosta \\ Department of Information and \\ Communication Technology, \\ Manipal Institute of Technology, \\ Manipal, India
}

\begin{abstract}
The main aim of this paper is to label a Self-Organizing Map (SOM) to measure image similarity. To achieve this, we input Facial images linked to the regions of interest into the neural network. Every single neural unit is tuned to a particular Facial image prototype at the end of the learning step. Probabilistic decision rule then performs facial recognition. This method gives accurate results for face identification taking into consideration various factors such as illumination variation and facial poses and expressions. The current research reveals a promising Self-Organizing Map (SOM) for face recognition. The Self-Organizing Map (SOM) technique is trained on images from one database. The novelty of this research comes from the integration of Images from input database, Training and Mapping. Face Recognition using unsupervised mode in neural network by Self-Organizing Map. Out of the all the architectures and algorithms suggested for artificial neural network, the SOM has an advantageous property of effectively creating a spatially organized 'internal representation' of various features of input signals and their abstractions. After the weight vectors have been finely tuned, the SOM technique has been promisingly accurate in various pattern recognition tasks involving very noisy signal. One develops realistic cortical structures when given approximations of visual environment as input, and is effective way to model the development of face recognition capability.

In this study Kohonen self-organizing map (SOM) based retrieval system has been used to develop and illustrate a recognition system for human faces. It is due to topological ordering that Self-organizing map possess a better and precise feature recognizing and extracting property. Factoring in the Facial Analysis solutions for over 400 images used from the AT\&T database we can say safely say that the facial recognition rate using one of the neural network algorithm SOM is $92.40 \%$ for 40 persons.
\end{abstract}

\section{Keywords}

SOM (Self Organizing Mapping), PCA (Principal Component Analysis), ICA (Independent Component Analysis).

\section{INTRODUCTION}

It is becoming increasingly difficult and extremely important to maintain the security of information or physical property in the present world. Credit card fraud, hacking of computers or security breaches in a company or government building have become very common in the current network world. It goes without saying that if someone steals duplicates. Modern technology has now enabled us to allow verification of "true" individual identity. This novel technology is based in a field called "biometrics "as shown in Fig. 2 and Fig. 3. High accuracy and low intrusiveness are the key merits possessed by facial detection method [5]. Accuracy of a physiological approach without being intrusive. Facial recognition is that it is a non-intrusive technique which can be effective without an individual's cooperation or knowledge which is the main reason for its great application in fields from security, surveillance and image processing. Several architectures and algorithms have been developed for facial recognition such as PCA, ICA [1][2].

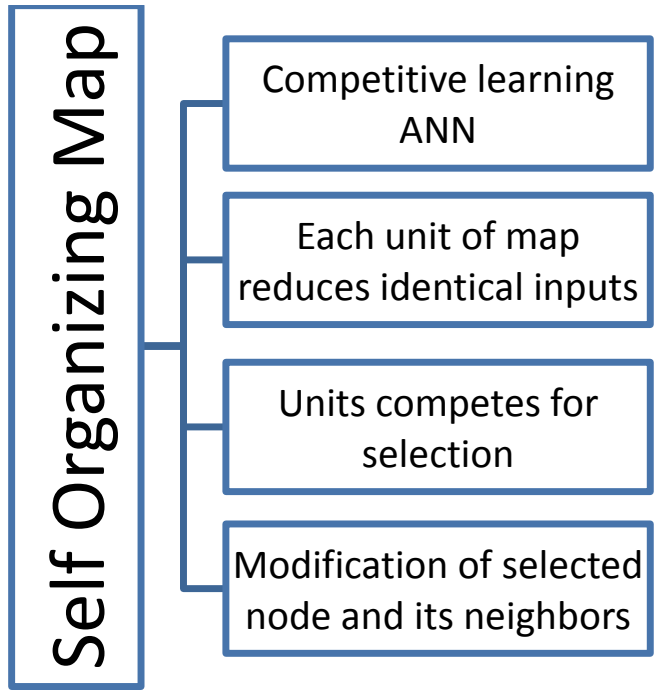

Fig. 1: Layout of a Self-Organizing Map.

The human capability to identify a particular individual by simply observing the human face is quite impressive. Changes in appearance and partial occlusion do not affect this human capability. Considerable attention has been paid to methods due to this remarkable ability to generate near-perfect positive identifications by which accurate face recognition can be replicated on an electronic level. Security and identity theft could be vastly improved in fields such as airport and bank and even private sectors if the immensely complicated task of identification of a person based on a technique as noninvasive as face recognition could be electronically achieved. 


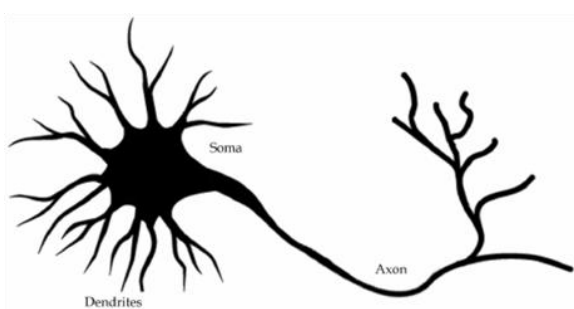

Fig. 2: Neuron Cell

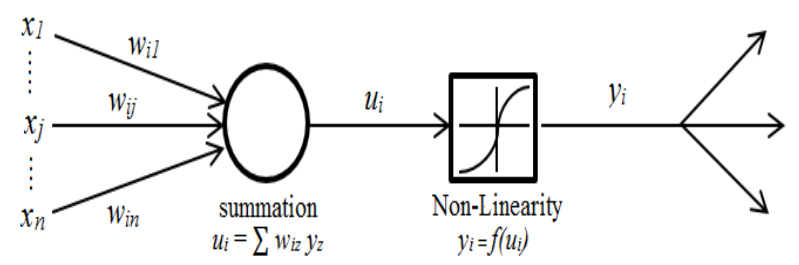

Fig 3:Neuron Model.

\section{CHALLENGES IN FACIAL RECOGNITION}

The major challenge in facial recognition is to Classification of Face Recognition first. Since to recognize a face a human should have knowledge about the face from earlier or at least must have seen the face before. Similarly, the facial recognition software needs a database to store the faces and need to verify the human's details. Thus, face recognition scenarios can be classified into two types, Face verification and Face identification.

a) Face verification: It is a one-to-one type of match in which a query face image is compared against a template face image whose identity is being claimed. To assess the verification performance, the verification rate (the rate, at which legitimate users are granted access) vs. false acceptance rate (the rate at which imposters are granted access) is strategized, called ROC curve. A worthy verification system should balance these two rates based on operational needs.

b) Face identification: It is a one-to-many type of matching process in which a query face image is compared against all the template images in a face database to conclude the identity of the query face. The identification of the test image is done by locating the image in the database that has the highest similarity with the test image [4].

The recognition process is a "closed" check, which means the sensor takes an observation of an individual that is known to be in the database. The test subject's characteristics are equated to the other characteristics in the system's database and a match score is found for each comparison. These match score are then numerically ranked in a descending order. The percentage of times that the highest match score is the correct match for all faces is stated to as the "top match score".

\section{REQUIREMENT ANALYSIS}

\subsection{Hardware Requirement}

a) $\quad s$ Computer System: Intel CPU @ $2.93 \mathrm{GHz}$ with i7 core, 4GB DDR2 RAM, G41 Gigabit Motherboard, On Board Soundcard Card \& AGP Card, 500GB
HDD, Cabinet with SMPS loaded with Operating System 8/Windows 10

b) Webcam, Scanner

\subsection{Software Requirements}

a) Matlab 7.8, Matlab 2008, Image Processing Toolbox

b) Outsourced Database

c) AT\&T Image Database

\section{SOM ALGORITHM AND} FLOWCHART

\subsection{Algorithm Steps.}

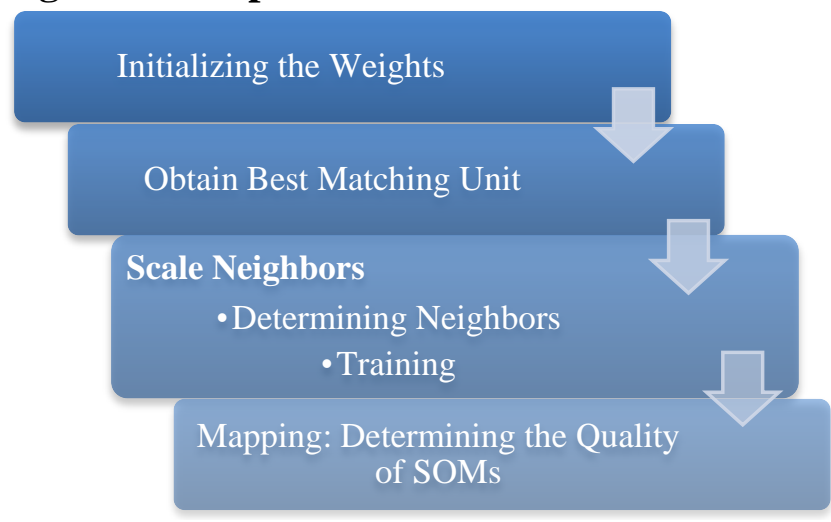

Fig. 4: Algorithm Steps

\subsection{Flow Chart}

Flow chart consists of two steps training and testing.
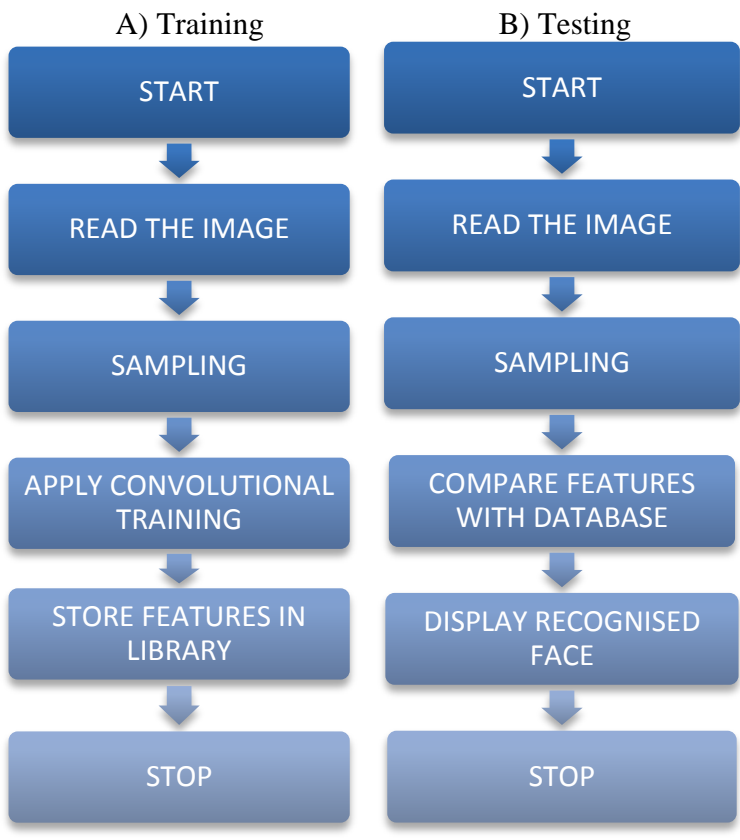

Fig. 5: Flow Chart for Face Recognition 


\section{INTRODUCTION TO THE SOFTWARE USED \\ 5.1. Overview of the MATLAB Environment}

The name MATLAB stands for matrix laboratory, initially written to give ease of access to matrix software made by the LINPACK and EISPACK projects. Nowadays, MATLAB engines also include the LAPACK and BLAS libraries, implanting the state of the art in software for matrix computation Fig. 6 .

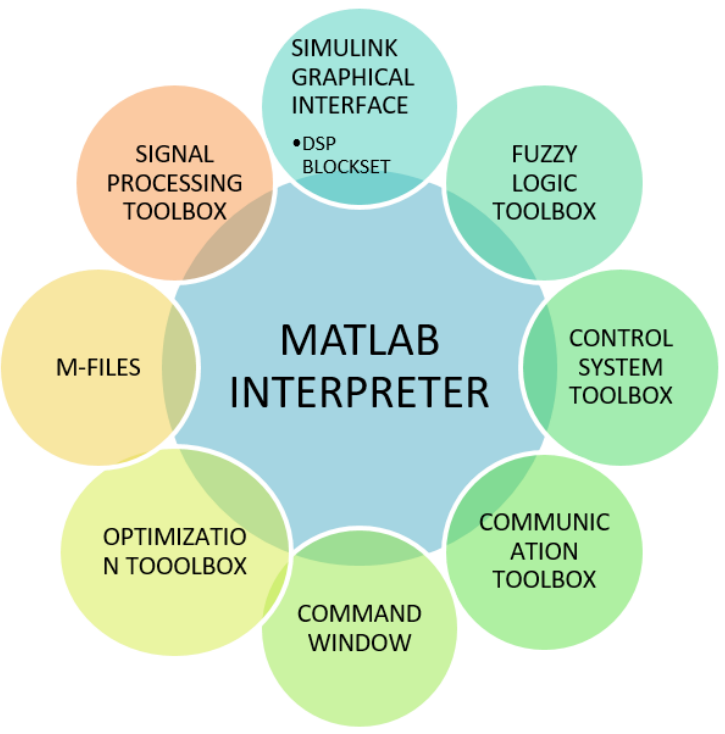

Fig. 6: Environment of MATLAB

\section{EXPERIMENTAL DATASET}

The AT\&T database is publicly available [6] and is used for a wide variety of facial recognition experiments. The database consists of a total of total 400 images (10 different images of each of 40 persons with a slight variation in conditions such as face angles, facial expressions and facial details. A preview image of the Database of Faces is as shown in Fig 7.

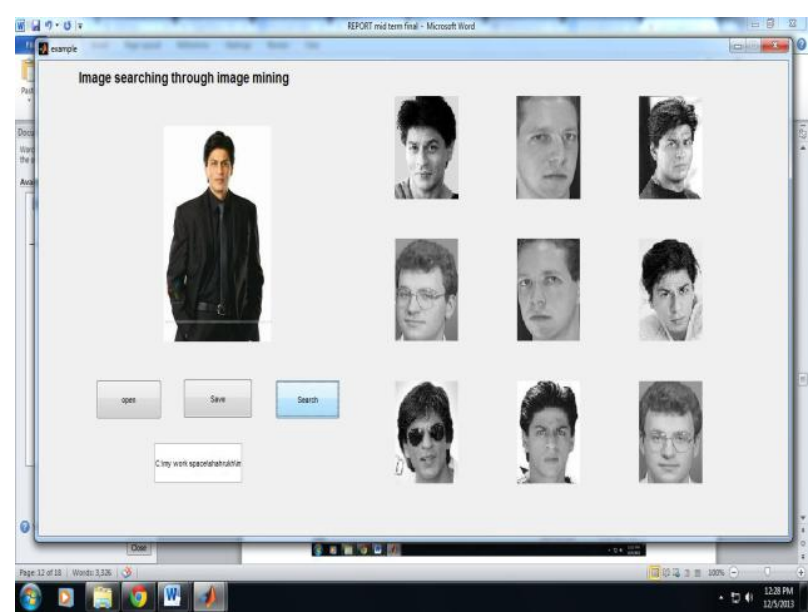

Fig. 7: AT\&T Dataset

\section{RESULTS}

Computational Efficiency of the SOM Method is as shown in Table 1 and Fig. 8.

Table 1: Result of SOM on ORL database

\begin{tabular}{|l|l|l|}
\hline SRN & DB IMAGE & $\begin{array}{l}\text { ACCURACY } \\
(\text { RECOGNITION } \\
\text { RATE })\end{array}$ \\
\hline $\mathbf{1}$ & 3 & $81.81 \%$ \\
\hline $\mathbf{2}$ & 4 & $83.72 \%$ \\
\hline $\mathbf{3}$ & 5 & $86.01 \%$ \\
\hline $\mathbf{4}$ & 6 & $92.20 \%$ \\
\hline $\mathbf{5}$ & 7 & $92.41 \%$ \\
\hline
\end{tabular}

\section{APPLICATIONS}

The main merit of facial recognition is that it is a nonintrusive technique which can be effective without an individual's cooperation or knowledge. This makes facial recognition suitable for surveillance purposes. Facial recognition techniques have been implemented by ATR (automatic target recognition), human traffic census security $\&$ Criminal identification uses.

Fig 8: Accuracy Vs People in Database

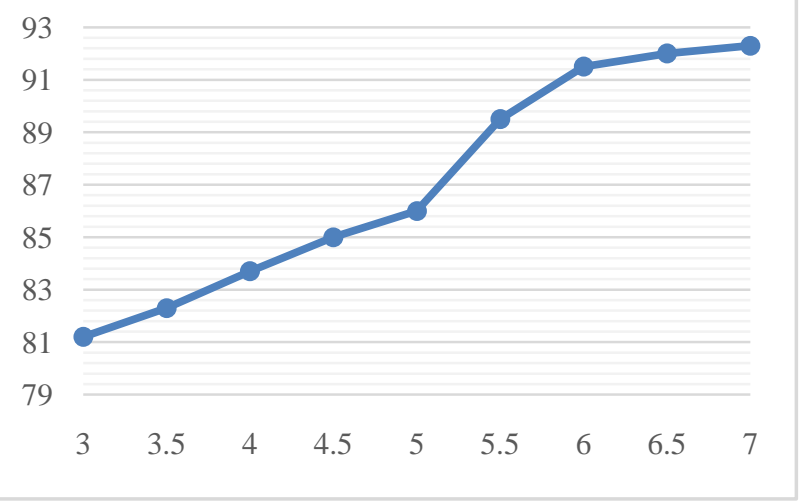

\section{CONCLUSION}

We have seen a Self-Organizing Map (SOM) method for face recognition. The innovation of this research comes from incorporation of input image, Feature extraction, Training and Mapping. Self-Organizing Mapping is sheet-like artificial neural network, the cells of which become specially accustomed to the different input signal patterns through an unsupervised learning process. Each cell behaves as a different decoder for the same input it is therefore the presence or absence of an active response at the location and not the exact magnitude of the input-output signal transformation, that gives an interpretation of the input information. SOM reduce dimensions and shows similarities. Since Self-Organizing Maps are topologically ordered, it possess a better and precise feature recognizing and extracting property. The highest average face recognition rate achieved using one of the neural network algorithms SOM is $92.40 \%$ 
for 40 persons. Thus our facial analysis results conclude that the complexity of a facial identification or recognition system decreases radically by using SOM.

\section{REFERENCES}

[1] G. B. Huang, M. Ramesh, T. Berg and E. LearnedMiller, "Labeled faces in the wild: A database for studying face recognition in unconstrained environments", 2007.

[2] R. Beveridge and H. Zhang, "Report on the FG 2015 video person recognition evaluation", Proc. IEEE Int. Conf. Autom. Face Gesture Recog., pp. 1-8, 2015.

[3] C. Ding and D. Tao, "A comprehensive survey on poseinvariant face recognition,"arXiv preprint arXiv:1502.04383, 2015.

[4] Santaji Ghorpade, Jayshree G., Shamla Mantri. (Dec 2010): Pattern Recognition using Neural Networks. IJCSIT, ISSN- 0975-9646, Vol 2, No. 6, pp92-98.
[5] Santaji Ghorpade, Jayshree G., Shamla Mantri, Dhanaji G. (Dec 2010): Neural Networks for Face Recognition using SOM.IJCST, ISSN-0976-8491, Vol 1, issue 2.

[6] Teuvo Kohonen.( 1990), Self-Organizing Map, Vol. 78, No.9.

[7] Dinesh Kumar Dimensionality. (May 2008), Reduction using SOM based on Technique for Face Recognition, Journal of Multimedia, Vol.3, No.1.

[8] F, Nazish Jamil, Fatima Jinnah Women University. Rewalpindi, Pakistan ace Recognition using Neural Networks.

[9] AT\&T Laboratories Cambridge, The database of faces at http://www.cl.cam.ac.uk/research/dtg/attarchive/facesata glance. html. 